\title{
Maternal death audit at tertiary care centre: changing trends
}

\author{
Sudha Sivakumar*, Sophia G. Xavarina, Ashmitaa Srianand
}

Department of Obstetrics and Gynecology, Madurai Medical College, Madurai, Tamil Nadu, India

Received: 30 January 2021

Revised: 17 February 2021

Accepted: 18 February 2021

\section{*Correspondence:}

Dr. Sudha Sivakumar,

E-mail: drsudha.sivakumar@gmail.com

Copyright: (c) the author(s), publisher and licensee Medip Academy. This is an open-access article distributed under the terms of the Creative Commons Attribution Non-Commercial License, which permits unrestricted non-commercial use, distribution, and reproduction in any medium, provided the original work is properly cited.

\begin{abstract}
Background: The aim of the study was to analyse the trend in maternal mortality ratio in a tertiary care centre.

Methods: This was a four-year retrospective analysis of maternal deaths that occurred at Government Rajaji Hospital, Department of Obstetrics and Gynaecology, Madurai from January 2017 to December 2020. Cases were stratified based on cause of death. Epidemiological data was collected from the hospital register.

Results: The total number of maternal deaths at GRH during the study period is 228 , in which death due to obstetric cause was $82(36 \%)$ and non-obstetric cause was $146(64 \%)$. The most common cause in each year was PIH (23\%2017, 29\%-2018), heart disease (22\%- 2019) and infection (46\%-2020).

Conclusions: We concluded by this study that the maternal death due to non-obstetric causes were more than the obstetric causes due to improved antenatal, post-natal care, institutionalized deliveries and timely referral to the CEmONC (comprehensive emergency obstetric and new-born care) centres and management.
\end{abstract}

Keywords: Haemorrhage, Heart disease, Maternal mortality ratio, Pregnancy induced hypertension, Sepsis

\section{INTRODUCTION}

Development of any nation depends on various factors, one of which is the ratio of maternal death to the total deliveries that year. Maternal death or maternal mortality is defined by the World Health Organization (WHO) as "the death of a woman while pregnant or within 42 days of termination of pregnancy, irrespective of the duration and site of the pregnancy, from any cause related to or aggravated by the pregnancy or its management but not from accidental or incidental causes". " Maternal deaths are further grouped based on the underlying cause of death such as direct and indirect causes and timing of death such as late maternal deaths. ${ }^{2}$ Maternal deaths are given importance as they reflect the growth of any society. They reflect the standard of health care in a community. They also determine the demographic advances in a country. Women in the least developed countries have on average many more pregnancies than women in developed countries, and their lifetime risk of death due to pregnancy is higher. Progress toward improvement in maternal health status are measured by useful maternal mortality indicators such as maternal mortality rate and ratio. Maternal mortality rate is the number of maternal deaths per 1000 women of reproductive age (usually 15-49 years) whereas maternal mortality ratio is the number of maternal deaths during a given time period per 100,000 live births during the same time period. This is more commonly used as it is better indicator of the burden of the disease. ${ }^{3}$

$$
\text { MMR }=\frac{\text { Total number of maternal deaths }}{\text { Total number of live births }} \times 100000
$$

Obstetric causes are due to obstetric complications, both unanticipated complications or from an inadequately managed complications during pregnancy, labour and the post-partum period. WHO estimates that the greatest proportion of maternal deaths are due to direct causes which include haemorrhage (25\%), sepsis (15\%), abortion $(13 \%)$, hypertensive disorders of pregnancy $(12 \%)$, and obstructed labour $(8 \%) .{ }^{4}$ Non-obstetric cause 
is due to other diseases that may exist before pregnancy, but is aggravated by the physiologic effects of pregnancy. About $20 \%$ of maternal deaths are due to indirect causes such as heart disease, iron deficiency anaemia, tuberculosis, hypertension, malaria, and diabetes mellitus. ${ }^{4}$

All women need access to high quality care provided by competent skilled health professionals during pregnancy, childbirth and in the weeks after childbirth. It is important that all deliveries are attended by skilled health professionals, as timely management and treatment can make the difference between life and death. Recent surveys show that the Institutional maternal mortality rates are 2-10 times higher because most of the seriously ill patients are referred to the tertiary care centre. ${ }^{5}$ Although many pregnant women are accessing the health services, lack of adequately trained personnel, bad referrals and delayed interventions account for most of the death. Therefore, identifying the cause of death and its incidence helps us to be more vigilant and anticipate adverse effects. They also help in forming new policies and widening our aspects of improved care of the patient. $^{6}$

Table 1: Total deliveries, death and MMR at Government Rajaji Hospital, Madurai.

\begin{tabular}{|lll|l|}
\hline Year & $\begin{array}{l}\text { Total } \\
\text { deliveries }\end{array}$ & $\begin{array}{l}\text { Maternal } \\
\text { deaths }\end{array}$ & $\begin{array}{l}\text { MMR/1,00,00 } \\
\text { live births }\end{array}$ \\
\hline $\mathbf{2 0 1 7}$ & 13142 & 81 & 616.34 \\
\hline $\mathbf{2 0 1 8}$ & 12721 & 55 & 432.35 \\
\hline $\mathbf{2 0 1 9}$ & 13767 & 41 & 297.81 \\
\hline $\mathbf{2 0 2 0}$ & 15869 & 51 & 321.38 \\
\hline Total & 55499 & 228 & 1667.88 \\
\hline
\end{tabular}

In this study, we tried to determine the maternal mortality ratio and the most common cause of deaths over the past four years and their trends so that we could scrutinize the existing protocol of management and point out improved strategies for an improved health care at our centre. We have also analysed the trends in the causes over the past four years.

\section{METHODS}

This was a retrospective study conducted at Government Rajaji Hospital of Madurai Medical College, a tertiary care centre which included all the maternal deaths that occurred from January 2017 to December 2020.

Our hospital has a 12 bedded maternal intensive care unit, 24 bedded obstetric high dependency unit, 12 bedded eclampsia unit with round the clock monitoring and critical care. 24 hours on call anesthetist team is available. Many cases are referred from nearby rural community health centres, public health centres, primary health centres, district hospital, civil hospitals, primary nursing homes.

\section{Inclusion criteria}

All maternal deaths occurring at our hospital for the past four years irrespective of their age, parity, socioeconomic status and their booking status were included.

\section{Exclusion criteria}

Patients who were brought dead were excluded.

Case sheets of all the 228 maternal deaths over the study period of four years was obtained from the Medical Record Department after getting permission from the Resident Medical Officer. Required details including cause of death was collected using a proforma as a study tool. The data was entered and analysed using Microsoft Excel Sheet 2019.

\section{RESULTS}

The total number of maternal mortalities for the year 2017 to 2020 was 228. Direct cause contributes to $36 \%$ and indirect cause contribute $64 \%$. Maternal mortality rate of India was 113 and of Tamil Nadu was 60 according to the 2018 bulletin. $^{4}$

Table 2: Obstetric versus non obstetric cause of MMR at Government Rajaji Hospital from 2017 to 2020.

\begin{tabular}{|lll|}
\hline Causes & No. of deaths & Percentage \\
\hline Obstetric & 82 & 35.96 \\
\hline Non obstetric & 146 & 64.04 \\
\hline
\end{tabular}

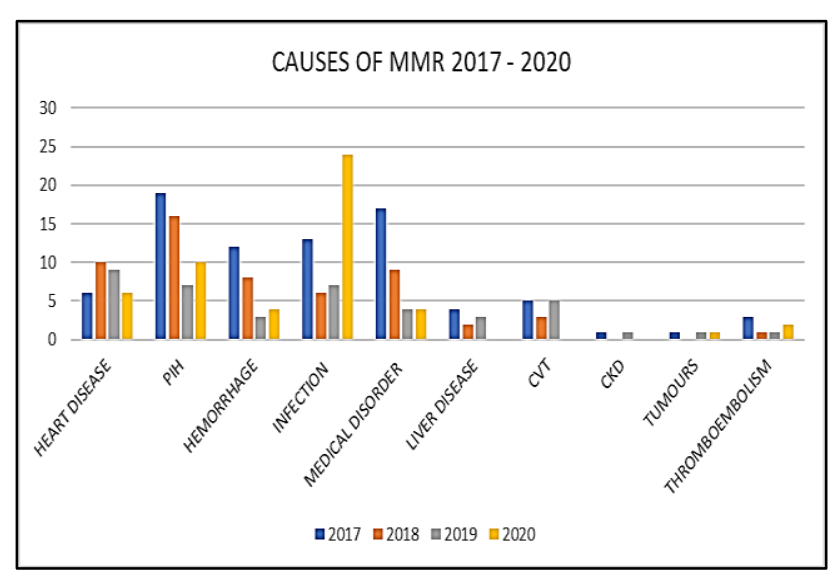

Figure 1: Causes of MMR at Government Rajaji Hospital from 2017 to 2020.

In this four-year study, maternal mortality due to hypertensive disorder $22.8 \%$, infection (including COVID deaths and infective endocarditis) $21 \%$, heart disease $13.6 \%$, postpartum haemorrhage $11.84 \%$, other medical causes $14.9 \%$, cortical venous thrombosis $5.7 \%$, pulmonary thromboembolism $3 \%$, liver disease $1.8 \%$, tumours $1.3 \%$, other obstetric cause $1.3 \%$ and chronic kidney disease $0.8 \%$. 
Table 3: Causes of MMR at Government Rajaji Hospital from 2017 to 2020.

\begin{tabular}{|lllll|}
\hline Heart disease & $\mathbf{2 0 1 7}$ & $\mathbf{2 0 1 8}$ & $\mathbf{2 0 1 9}$ & $\mathbf{2 0 2 0}$ \\
\hline PIH & $6(7.4 \%)$ & $10(18.2 \%)$ & $9(21.9 \%)$ & $6(11.76 \%)$ \\
\hline Hemorrhage & $19(23.5 \%)$ & $16(29.1 \%)$ & $7(17.07 \%)$ & $10(19.6 \%)$ \\
\hline Infection & $12(14.8 \%)$ & $8(14.5 \%)$ & $3(7.13 \%)$ & $4(7.84 \%)$ \\
\hline Medical disorder & $13(16.04 \%)$ & $6(10.9 \%)$ & $7(17.07 \%)$ & $24(47.05 \%)$ \\
\hline Liver disease & $17(20.99 \%)$ & $9(16.36 \%)$ & $4(10.90 \%)$ & $4(9.75 \%)$ \\
\hline CVT & $2(2.46 \%)$ & $1(1.81 \%)$ & $3(7.31 \%)$ & - \\
\hline CKD & $5(6.17 \%)$ & $3(5.45 \%)$ & $5(12.19 \%)$ & - \\
\hline Tumours & $1(1.23 \%)$ & - & $1(2.43 \%)$ & - \\
\hline Thromboembolism & $1(1.12 \%)$ & - & $1(2.43 \%)$ & $1(1.96 \%)$ \\
\hline Total & $3(3.70 \%)$ & $1(1.81 \%)$ & $1(2.43 \%)$ & $2(3.92 \%)$ \\
\hline
\end{tabular}

\section{DISCUSSION}

Being a predominantly referral hospital, most of the women coming have risk factors and many are in a moribund state. ${ }^{7}$ As such the maternal mortality ratio is always high in a tertiary care centre as compared to periphery. As per the latest report (2016-18) of sample registration system (SRS) released by Registrar General of India (RGI), Maternal Mortality Ratio (MMR) of India per 100,000 live births has declined to 113 in 2016-18 from 122 in 2015-17 and 130 in 2014-2016. ${ }^{8}$ Assam continues to be the state of highest MMR (229). ${ }^{9}$ Tamil Nadu's maternal mortality ratio has reduced from 90 per one lakh live births in 2010-2012 to 63 in 2015-2017 and further to 57 in 2019 as per data maintained by the State government. As per health management information systems (HMIS) data, the State's MMR stood at 60 per one lakh live births in $2018 .^{7}$ This decline to 57 per one lakh live births is a good sign and is proof that maternal care has improved in the State. ${ }^{10}$ India's share among global maternal death has declined significantly as per United nations maternal mortality estimation inter-agency group (UN- MMEIG) report. ${ }^{9}$

Table 4: MMR rates in India and Tamil Nadu from 2016 to 2018.

\begin{tabular}{|llll|}
\hline MMIR rates & $\mathbf{2 0 1 4 - 1 6}$ & $\mathbf{2 0 1 5 - 1 7}$ & $\mathbf{2 0 1 6 - 1 8}$ \\
\hline India & 130 & 122 & 113 \\
\hline Tamil Nadu & 66 & 63 & 60 \\
\hline
\end{tabular}

A single intervention alone has not brought about this decline. But a number of efforts, starting with the health of adolescent girls to ante-natal and post-natal care of mothers including high risk women has led to this further reduction. Comprehensive analyses of the causes of maternal mortality have been published by WHO and the Institute for Health Metrics and Evaluation. ${ }^{11,12}$ These analyses strikingly show the increasing importance of indirect causes of maternal death. Say and colleagues noted that $27.5 \%$ of all maternal deaths result from these indirect causes, with the highest proportion of such deaths in South Asia and Sub-Saharan Africa. ${ }^{11}$ Kassebaum and colleagues similarly show the growing direct and indirect effects of non-communicable diseases on maternal mortality. ${ }^{12}$ These authors conclude that indirect causes of maternal deaths cannot be ignored and that efforts should be focused on their reduction.

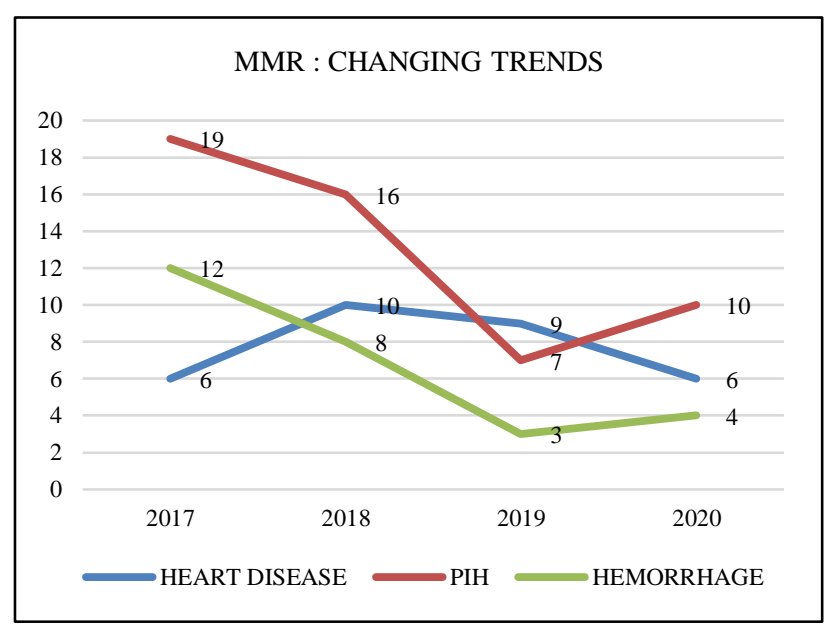

Figure 2: Changing trends in MMR at Government Rajaji Hospital (2017-2020).

Under the National Health Mission (NHM), some of the important steps taken to reduce maternal deaths in the country are as follows: Janani Suraksha Yojana (JSY), a demand promotion and conditional cash transfer scheme is implemented with the objective of reducing maternal and infant mortality by encouraging institutional deliveries. ${ }^{9,13}$ Under Janani Shishu Suraksha Karyakram (JSSK), every pregnant woman is entitled to free delivery, including caesarean section in public health institutions. This also includes free transport, diagnostics, medicines, other consumables, food and blood, if required. Pradhan Mantri Surakshit Matritva Abhiyan (PMSMA) has been launched to provide fixed-day assured comprehensive and quality antenatal care universally to all pregnant women on the $9^{\text {th }}$ of every month. While antenatal care is routinely provided to pregnant women, special ANC services are provided by 
OBGY specialists/ radiologist/ physicians at government health facilities under PMSMA. 'LaQshya - labour room quality improvement initiative' has been launched on $11^{\text {th }}$ Dec 2017. Maternal death surveillance and response (MDSR) is implemented to take corrective action for averting maternal deaths. Funds are provided for strengthening of 'delivery points' for provision of comprehensive reproductive, maternal, new born child health and adolescent ( $\mathrm{RMNCH}+\mathrm{A})$ services. Placing quality emergency obstetric care services at "delivery points" is a priority area. Maternal and child health (MCH) wings are established at high caseload facilities. Operationalization of comprehensive abortion care services and services for reproductive tract infections and sexually transmitted infections (RTI/STI). Mother and child tracking system (MCTS) and mother and child tracking facilitation centre (MCTFC), a name-based webenabled system to track every pregnant women and child. Operational guidelines for Universal screening of gestational diabetes mellitus, screening for hypothyroidism for high risk group during pregnancy, training of general surgeons for performing caesarean section, calcium supplementation and de-worming during pregnancy and promotion of birth companion during delivery have been disseminated. Monthly village health and nutrition days (VHND) are organized as an outreach activity at Anganwadi centres for provision of maternal and child care including nutrition. Over 10 lakhs accredited social health activists (ASHAs) have been engaged to facilitate access of healthcare services by the community, particularly pregnant women. Under the National Iron+ Initiative, iron and folic acid supplementation is given across life stages including pregnant, lactating women and adolescent girls. ${ }^{9}$ With all these diverse programs, the maternal mortality due to haemorrhage have shown a declining trend and now the focus has shifted to the early diagnosis and prevention of pregnancy induced hypertension and non-obstetric causes. One such initiative is the Tamil Nadu Pregnancy and Heart Disease Registry in which all the cardiac conditions complicating pregnancy are registered at the earliest visit and followed up till their 42 days of postpartum period.

However, there are some limitations in this study. The first one is the limited time period of the study. The next one is that only deaths that occurred in our tertiary care was taken in to account.

\section{CONCLUSION}

We concluded by this study that the cause of maternal death has shifted more towards indirect and non-obstetric causes. There is a declining trend in direct obstetric causes due to the improved antenatal, post-natal care, institutionalised deliveries and timely referral to the CEmONC (comprehensive emergency obstetric and newborn care) centres and management.
Funding: No funding sources

Conflict of interest: None declared

Ethical approval: The study was approved by the Institutional Ethics Committee

\section{REFERENCES}

1. World Health Data Platform. GHO, Indicator Metadata Registry List. Maternal mortality ratio (per $100 \quad 000$ live births). Available at: https://www.who.int/data/gho/indicator-metadataregistry/imr-details/26. Accessed on 4 October 2020.

2. Patwardhan M, Eckert LO, Spiegel H, Pourmalek F, Cutland C, Kochhar S, et al. Maternal death: Case definition and guidelines for data collection, analysis, and presentation of immunization safety data. Vaccine. 2016;34(49):6077.

3. Government of India. Special bulletin on maternal mortality in India 2016-18: Sample registration system. Government of India. Retrieved September 24, 2020. Available at: https://censusindia.gov.in/vital_statistics/SRS_Bullet ins/MMR\%20Bulletin\%202016-18.pdf. Accessed on 10 October 2020.

4. WHO, UNICEF, UNFPA, World Bank, United Nations. Trends in maternal mortality: 1990 to 2013. Geneva: World Health Organization; 2014.

5. Kulkarni Sunanda R, Huligol A, Maternal mortality: 10 years study. J Obstet Gynaecol India. 2001;51:736.

6. Shrivastav S. Maternal mortality rate among worst in India, TNN. 2011.

7. Bedi N, Kambo I, Dhillon BS, Saxena BN, Singh P. Maternal deaths in India- preventable tragedies (An ICMR-Task Force Study. J Obstet Gynaecol India. 2001;51:86-92.

8. SRS Bulletin- MMR. Available at: https://censusindia.gov.in/vital_statistics/SRS_Bullet ins/MMR_Bulletin-2015-17.pdf. Accessed on 10 October 2020.

9. Department of health and family welfare, Government of India. Maternal and adolescent healthcare. Annual Report 2019-2020. 2020;25.

10. Ministry of Women and Child Development/ Reduction in maternal mortality. Available at: https://pib.gov.in/PressReleaseIframePage.aspx?PRI $\mathrm{D}=1656242$. Accessed on 10 October 2020.

11. Say L, Chou D, Gemmill A, Tunçalp Ö, Moller AB, Daniels J, et al. Global causes of maternal death: a WHO systematic analysis. Lancet Glob Health. 2014;2(6):e323-33.

12. Kassebaum NJ, Bertozzi-Villa A, Coggeshall MS, Shackelford KA, Steiner C, Heuton KR, et al. Global, regional, and national levels and causes of maternal mortality during 1990-2013: a systematic analysis for the Global Burden of Disease Study 2013. Lancet. 2014;384(9947):980-1004.

13. Lim SS, Dandona L, Hoisington JA, James SL, Hogan MC, Gakidou E. India's Janani Suraksha Yojana, a conditional cash transfer programme to 
increase births in health facilities: an impact evaluation. Lancet. 2010;375(9730):2009-23.

14. Dutta DK. Strategies to reduce maternal mortality and morbidity in Rural India. The Federation of Obstetrics and Gynecological Society of India. Available at: https://www.fogsi.org/strategies-to- reduce-maternal-mortality-and-morbidity-in-ruralindia/. Accessed on 10 October 2020.

Cite this article as: Sivakumar S, Xavarina SG, Srianand A. Maternal death audit at tertiary care centre: changing trends. Int J Reprod Contracept Obstet Gynecol 2021;10:915-9. 